\title{
Effects of treadmill exercise on activity, short-term memory, vascular dysfunction in maternal separation rats
}

\author{
Sang-Seo Park', Tae-Woon Kim²,2, Hye-Sang Park ${ }^{3}$, Tae-Beom Seo ${ }^{4}$, Young-Pyo Kim ${ }^{4, *}$ \\ 'Department of Physiology, College of Medicine, Kyung Hee University, Seoul, Korea \\ ${ }^{2}$ Exercise Rehabilitation Research Institute, Department of Exercise \& Health Science, Sangmyung University, Seoul, Korea \\ ${ }^{3}$ Department of Kinesiology, College of Public Health and Cardiovascular Research Center, Lewis Katz School of Medicine, Temple University, Philadelphia, PA, USA \\ ${ }^{4}$ Department of Kinesiology, College of Natural Sciences, Jeju National University, Jeju, Korea
}

Maternal separation during early life causes psychiatric and neurological disorders such as anxiety and depression. Depression or anxiety is closely associated with memory impairment. The purpose of this study was to investigate the effect of treadmill exercise on activity, short-term memory, vascular dysfunction using maternal separation-induced depression model. Maternal separation started on 15-day-old rats. The rats in the maternal separation and fluoxetine injection group received intraperitoneal injection of $5 \mathrm{mg} / \mathrm{kg}$ of fluoxetine one time daily for 15 days from 21 to 35 days. The rats performed treadmill exercise once a day during 15 days from 21 to 35 days. There was low activity and shortterm memory was decreased in the maternal separation rats. Treadmill exercise and fluoxetine injection increased activity and ameliorated memory impairment. The number of rat endothelial cells antigen-1 (RECA-1) of microvessels was decreased in the maternal separation rats. The number of RECA-1was increased by treadmill exercise and fluoxetine injection. Expression of matrix metalloproteinase-9 (MMP-9) was increased and expressions of zonula occludens-2 (ZO-2) and occludin were decreased in the maternal separation rats. Treadmill exercise and fluoxetine injection suppressed MMP-9 expression and enhanced ZO-2 and occludin expressions in the maternal separation rats. The present study shows treadmill exercise and antidepressant treatment ameliorates depressive symptom and short-term memory impairment by protecting from blood-brain barrier damage.

Keywords: Maternal separation, Short-term memory, Treadmill exercise, Rat endothelial cells antigen-1, Matrix metalloproteinase-9, Zonula occludens-2

\section{INTRODUCTION}

Maternal separation during early life causes psychiatric and neurological disorders, such as anxiety and depression (Kalinichev et al., 2002). Patients with vascular dementia have a significantly higher incidence of depression than patients with Alzheimer disease (Srikanth et al., 2005). The relationship between vascular disease and depression has multifactorial etiologies, such as homocysteine, cytokines, and endothelial dysfunction (Teper and O'Brien, 2008). Depression or anxiety is closely associated with memory impairment (Park et al., 2019).

Vascular dementia is associated with dysfunction of the bloodbrain barrier (BBB). BBB exists between the brain and blood ves-

sels and prevents certain substances from entering the brain through the blood vessels. The BBB is critical for brain homeostasis and located at the cerebral microvessel endothelial cells. There are two types antibodies against rat endothelial cells antigen (RECA): monoclonal antibodies to RECA-1 and RECA-2. Antibody to RECA-1 is a promising antibody for rat endothelial cell studies, and in particular for further defining nature and function of endothelial cell-specific antigens. These endothelial cells maintain their barrier characteristics via cell-cell contacts made up of adherens and tight junctions. Adherens junctions are calcium dependent, and recent evidence suggests that calcium also affects tight junctions (Brown and Davis, 2002).

The BBB is composed of membrane proteins, including tight

${ }^{*}$ Corresponding author: Young-Pyo Kim (iD https://orcid.org/0000-0001-6662-1393 Department of Kinesiology, College of Natural Science, Jeju Natioanal University, 102 Jejudaehak-ro, Jeju 63243, Korea

E-mail: kimyp@jejunu.ac.kr

Received: February 7, 2020 / Accepted: April 1, 2020 
junction proteins such as occludin, claudin, and junctional adhesion molecules, and auxiliary proteins, such as zonula occludens (ZO) (Hawkins and Davis, 2005). ZO-1 and ZO-2 are cytosolic scaffolds that regulate the assembly of cellular junctions. Occludin is an enzyme that oxidizes nicotinamide adenine dinucleotide phosphate (Castro et al., 2016). Occludin is important in tight junction stability and barrier function.

Vascular disease results in the destruction of the basement membrane of the cerebrovascular vesels, a component of BBB (Zlokovic, 2010). The increase of BBB permeability due to vascular dysfunction is caused by increased activity of the matrix metalloproteinase-9 (MMP-9), a degradation protein present in the extracellular matrix. Increase in the MMP-9 level causes increased permeability and destruction of BBB (Yang and Rosenberg, 2011).

Selective serotonin reuptakes in inhibitors (SSRIs) have been used to alleviate depressive symptom. SSRIs enhance neurotransmission by allowing serotonin to act longer by blocking presynaptic reuptake mechanisms (Pittenger et al., 2008). Exercise is known to have a neuroprotective effect on various brain injuries (Cotman and Engesser-Cesar, 2002). Physical activity exerts protective effect on depression (Strawbridge et al., 2002). The purpose of this study was to investigate the effect of treadmill exercise on activity, short-term memory, vascular dysfunction using maternal separation-induced depression model.

\section{MATERIALS AND METHODS}

\section{Experiment animals}

This study obtained approval by the Institutional Animal Care and Use Committee of Kyung Hee University (KHUASP [SE]16-108). Sprague-Dawley rats were randomly divided into five groups ( $\mathrm{n}=8$ per group): control group, control and exercise group, maternal separation group, maternal separation and exercise group, and maternal separation and fluoxetine injection group.

Maternal separation started on 15-day-old rats. The rats in the control group and in the control and exercise group were lived with their mothers. The rats in the maternal separation and fluoxetine injection group received intraperitoneal injection of $5 \mathrm{mg} / \mathrm{kg}$ of fluoxetine one time daily for 15 days from 21 to 35 days.

\section{Exercise protocol}

According to the previous study (Lee et al., 2017), the animals in exercise groups were made to run on treadmill once a day during 15 days from 21 to 35 days. The exercise load composed of warm up during $5 \mathrm{~min}$ at $2 \mathrm{~m} / \mathrm{min}$, main exercise during $30 \mathrm{~min}$ at
$5 \mathrm{~m} / \mathrm{min}$, and cool down during $5 \mathrm{~min}$ at $2 \mathrm{~m} / \mathrm{min}$ with a 0 inclination.

\section{Open field test}

To determine the activity of the rats, open field test was conducted, as a previously described method (Park et al., 2019). The rats were randomly assigned to an order of testing and placed in a white square open field arena $(100 \mathrm{~cm} \times 100 \mathrm{~cm})$ made of wood, enclosed by $40-\mathrm{cm}$-high walls and exposed to strong illumination (200 lux). The arena was divided into 16 squares $(20 \mathrm{~cm} \times 20 \mathrm{~cm})$, consisting of 4 central and 12 peripheral squares. The rats were placed in the center of the arena and were allowed to explore the environment for $1 \mathrm{~min}$. Following this time, the number of squares crossed was recorded for $5 \mathrm{~min}$.

\section{Step-down avoidance test}

Short-term memory was determined using the step-down avoidance test, according to the previously study (Park et al., 2019). On the 15 days after starting exercise, the rats were trained. In the training session, the rats were placed on a $7 \times 25-\mathrm{cm}$ platform, $2.5 \mathrm{~cm}$ in height. The platform faced a $42 \times 25-\mathrm{cm}$ grid of parallel, $0.1 \mathrm{~cm}$ caliber stainless steel bars spaced $1 \mathrm{~cm}$ apart. The rats received electrical foot shock $(0.2 \mathrm{~mA})$ for 2 sec immediately upon stepping down. One day after training session, the latency (sec) of the rats was determined. Latency was the time interval of rats stepping down from platform and placing all four paws on the grid. Over $300 \mathrm{sec}$ in latency was counted as $300 \mathrm{sec}$.

\section{Tissue preparation}

The rats were sacrificed immediately after determination of step-down avoidance test. To prepare the brain slices, the rats were fully anesthetized with ethyl ether after which the rats were transcardially perfused with $50 \mathrm{mM}$ phosphate-buffered saline and then fixed with freshly prepared $4 \%$ paraformaldehyde in $100 \mathrm{mM}$ phosphate buffer $(\mathrm{pH}, 7.4)$. After the brains were removed, postfixed in the same fixative overnight, and transferred into a $30 \%$ sucrose solution for cryoprotection. Coronal sections with thicknesses of $40 \mu \mathrm{m}$ were made using a freezing microtome (Leica, Nussloch, Germany).

\section{Immunohistochemistry for RECA-1}

To visualize endothelial microvessels expression, immunohistochemistry for RECA-1 in the hippocampal dentate gyrus was performed, as a previously described method (Lee et al., 2017). After being blocked with $10 \% \mathrm{hr}$ and rabbit serum for $1 \mathrm{hr} 30 \mathrm{~min}$, the 



Fig. 1. Activity in open field test and short-term memory in step-down avoidance test. A, control group; B, control and exercise group; C, maternal separation group; D, maternal separation and exercise group; $E$, maternal separation and fluoxetine treatment group. The data are presented as the mean \pm standard error of the mean. ${ }^{*} P<0.05$ compared to the control group. ${ }^{*} P<0.05$ compared to the maternal separation group.

sections were incubated overnight at $4^{\circ} \mathrm{C}$ with RECA-1 antibody (1:1,000; Abcam, Cambridge, UK). The sections were then incubated for $2 \mathrm{hr}$ with the biotinylated mouse secondary antibody (1:200; Vector Laboratories, Burlingame, CA, USA). The secondary antibody was then amplified using a Vector Elite $\mathrm{ABC}$ kit (1: 100; Vector Laboratories). The antibody-biotin-avidin-peroxidase complex was visualized using $0.02 \%$ diaminobenzidine. The slides were air-dried overnight at room temperature, and the coverslips were mounted using Permount (Fisher Scientific, Pittsburgh, PA, USA).

\section{Western blotting for MMP-9, ZO-2, and occludin}

Western blotting was performed, as a previously described method (Lee et al., 2017). The hippocampus tissues were homogenized on ice and lysed in a lysis buffer containing $50 \mathrm{mM}$ Tris- $\mathrm{HCl}(\mathrm{pH}$, 7.5), $150 \mathrm{mM} \mathrm{NaCl}, 0.5 \%$ deoxycholic acid, $1 \%$ Nonidet P40, $0.1 \%$ sodium dodecyl sulfate (SDS), $1 \mathrm{mM}$ phenylmethylsulfonyl fluoride, and 100-mg/mL leupeptin. Protein content was measured using a Bio-Rad colorimetric protein assay kit (Bio-Rad, Hercules, CA, USA). Protein of $40 \mu \mathrm{g}$ was separated on SDS-polyacrylamide gels and transferred onto a nitrocellulose membrane, which was incubated with mouse $\beta$-actin antibody (1:1,000; Santa Cruz Biotechnology, Santa Cruz, CA, USA), rabbit MMP-9 antibody (1: 1,000; Santa Cruz Biotechnology), ZO-2 antibody (1:1,000; Santa Cruz Biotechnology) and occludin antibody (1:1,000, Santa Cruz Biotechnology). Horseradish peroxidase-conjugated anti-mouse for $\beta$-actin, and anti-rabbit for MMP-9, ZO-2, and occludin were used as secondary antibodies.
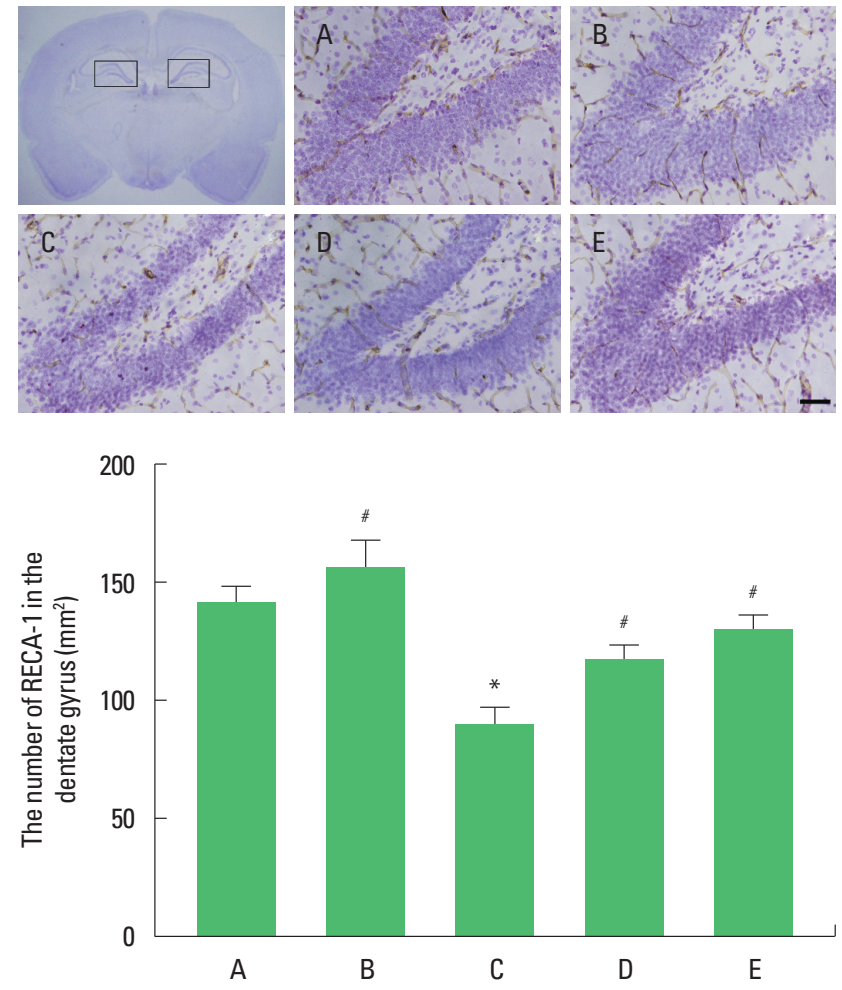

Fig. 2. Endothelial microvessels in the hippocampal dentate gyrus. Upper panel: Photomicrographs of rat endothelial cell antigen-1 (RECA-1)-positive endothelial microvessels. Lower panel: The number of RECA-1-positive endothelial microvessels in each group. The scale bar represents $500 \mu \mathrm{m}$ in each group. $A$, control group; $B$, control and exercise group; $C$, maternal separation group; $D$, maternal separation and exercise group; $\mathrm{E}$, maternal separation and fluoxetine treatment group. The data are presented as the mean \pm standard error of the mean. ${ }^{*} P<0.05$ compared to the control group. ${ }^{\sharp} P<0.05$ compared to the maternal separation group. 


\section{Data analysis}

The number of REAC-1-positive cells in hippocampal dentate gyrus was counted hemilaterally under a light microscope (Olympus, Tokyo, Japan). For confirming the expressions of MMP-9, ZO-2, and occludin, the detected bands were calculated densitometrically using Image-Pro Plus software (Media Cybernetics, Silver Spring, MD, USA). The data were analyzed with one-way analysis of variance by Tukey post hoc test. All results were expressed as the mean \pm standard error of the mean, and $P$-value of $<0.05$ was considered significant.

\section{RESULTS}

\section{Activity and short-term memory}

The average distance in center on the open field test is presented in Fig. 1 (left panel). The rats in the maternal separation group showed decreased activity compared to the control group. In contrast, treadmill exercise and fluoxetine injection increased activity in the maternal separation rats.

The latency time in the step-down avoidance test is presented in Fig. 1 (right panel). The rats in the maternal separation group showed impairment of short-term memory compared to the control group. In contrast, treadmill exercise and fluoxetine injection alleviated short-term memory impairment in the maternal separation rats.

\section{Endothelial microvessels in the hippocampal dentate gyrus}

Photomicrographs of RECA-1 of microvessels in hippocampal dentate gyrus are presented in Fig. 2. The number of RECA-1-positive microvessels was decreased in the maternal separation group compared to the control group. In contrast, treadmill exercise and fluoxetine injection increased the number of RECA-1-positive in the maternal separation rats.

\section{Expressions MMP-9, ZO-2, and occludin in the hippocampus}

Expressions of MMP-9, ZO-2, and occludin in the hippocampus are presented in Fig. 3. MMP-9 expression was increased in in the maternal separation group compared to the control group. In contrast, treadmill exercise and fluoxetine injection decreased MMP-9 expression in the maternal separation rats (Fig. 3, lower left panel).

ZO-2 expression was decreased in in the maternal separation group compared to the control group. In contrast, treadmill exercise and fluoxetine injection increased ZO-2 expression in the maternal separation rats (Fig. 3, lower middle panel).

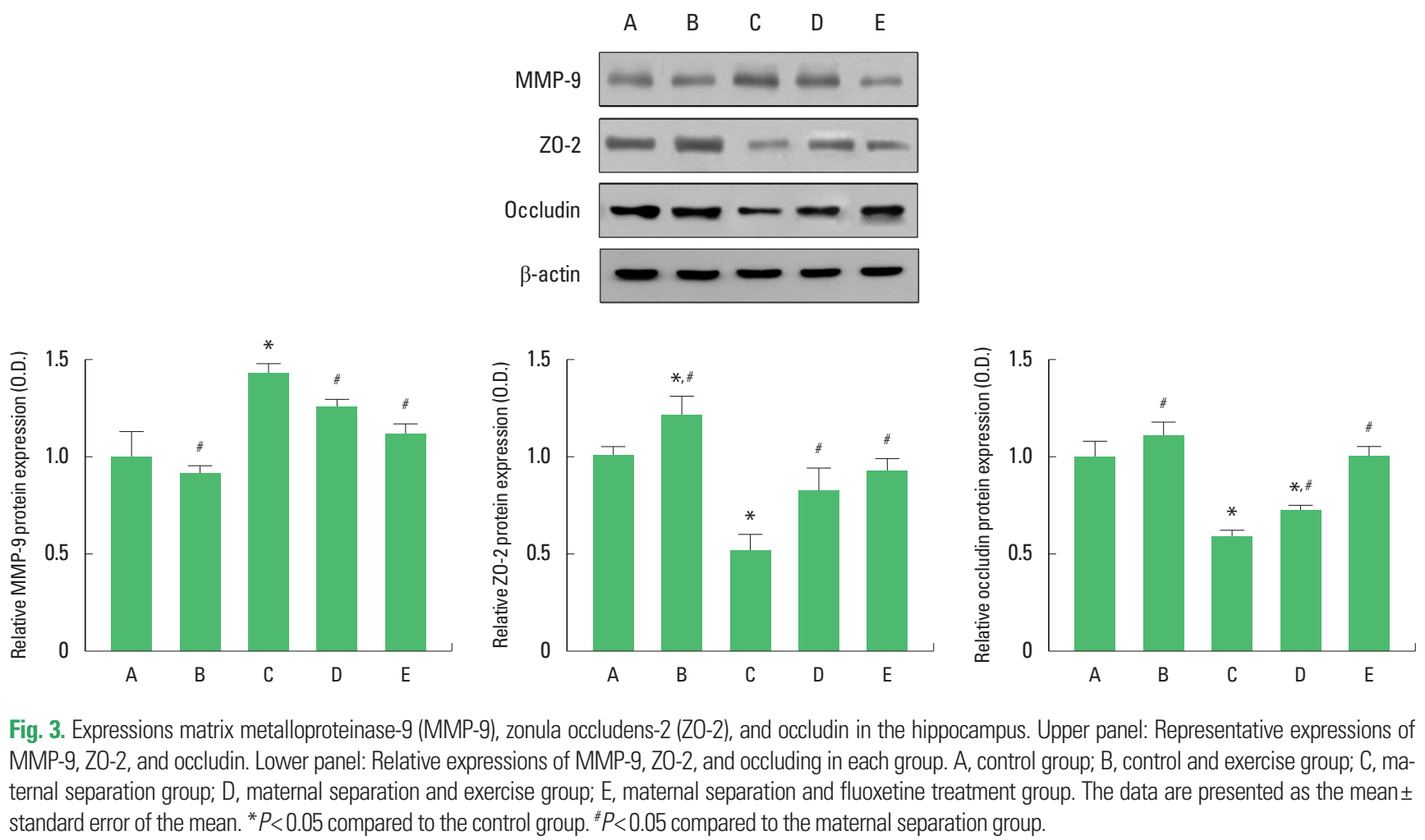


Occludin expression was decreased in in the maternal separation group compared to the control group. In contrast, treadmill exercise and fluoxetine injection increased occludin expression in the maternal separation rats (Fig. 3, lower right panel).

\section{DISCUSSION}

Rats that experienced maternal separation showed depressivelike behavior and cognitive impairment (Aisa et al., 2008). Desbonnet et al. (2010) reported that a long-term separation from the mother caused a significant amount of stress. In this study, open field test was conducted to determine the degree of activity. There was low activity in the rats of the maternal separation group, demonstrating that maternal separation-induced depressive symptom. Step-down avoidance test was performed to measure short-term memory. Short-term memory was decreased in the maternal separation rats. Treadmill exercise and fluoxetine injection increased activity and ameliorated memory impairment.

There is a growing list of brain pathologies showing BBB dysfunction, with strong evidence that BBB dysfunction plays a major role in certain disease etiology (Abbott, 2012). Dysfunction of the vascular unit with BBB and permeability are caused by major depressive disorders (Najjar et al., 2013). In this study, the number of RECA-1 of microvessels was decreased in the maternal separation rats. The number of RECA-1was increased by treadmill exercise and fluoxetine injection.

Proteins that made up tight junctions include ZO-2 and occludin (Hawkins and Davis, 2005). Chronic cerebral hypoperfusion induced disruption of BBB. Degradation of occludin, ZO-1, and up-regulation of MMP-9 were appeared in disruption of BBB (Lee et al., 2017). Treadmill exercise exerted protective effects on BBB disruption from degradation of occludin, ZO-1, and overexpression of MMP-9 after chronic cerebral hypoperfusion. These findings suggest that treadmill exercise ameliorated cognitive impairment and white matter injury from BBB disruption (Lee et al., 2017). In this study, expression of MMP-9 was increased and expressions of ZO-2 and occluding were decreased in the maternal separation rats. Treadmill exercise and fluoxetine injection suppressed MMP-9 expression and enhanced ZO-2 and occluding expression in the maternal separation rats.

The present study shows treadmill exercise and antidepressant treatment ameliorates depressive symptom and short-term memory impairment by protecting from BBB damage. There was no difference in treatment effectiveness between treadmill exercise and antidepressant.

\section{CONFLICT OF INTEREST}

No potential conflict of interest relevant to this article was reported.

\section{ACKNOWLEDGMENTS}

This work was supported by the Ministry of Education of the Republic of Korea and the National Research Foundation of Korea (NRF-2017S1A5A2A01027220). This research was supported by the 2019 Scientific Promotion Program funded by Jeju National University.

\section{REFERENCES}

Abbott NJ, Friedman A. Overview and introduction: the blood-brain barrier in health and disease. Epilepsia 2012;53 Suppl 6:1-6.

Aisa B, Tordera R, Lasheras B, Del Río J, Ramírez MJ. Effects of maternal separation on hypothalamic-pituitary-adrenal responses, cognition and vulnerability to stress in adult female rats. Neuroscience 2008; 154:1218-1226.

Brown RC, Davis TP. Calcium modulation of adherens and tight junction function: a potential mechanism for blood-brain barrier disruption after stroke. Stroke 2002;33:1706-1711.

Castro V, Bertrand L, Luethen M, Dabrowski S, Lombardi J, Morgan L, Sharova N, Stevenson M, Blasig IE, Toborek M. Occludin controls HIV transcription in brain pericytes via regulation of SIRT-1 activation. FASEB J 2016;30:1234-1246.

Cotman CW, Engesser-Cesar C. Exercise enhances and protects brain function. Exerc Sport Sci Rev 2002;30:75-79.

Desbonnet L, Garrett L, Clarke G, Kiely B, Cryan JF, Dinan TG. Effects of the probiotic Bifidobacterium infantis in the maternal separation model of depression. Neuroscience 2010;170:1179-1188.

Hawkins BT, Davis TP. The blood-brain barrier/neurovascular unit in health and disease. Pharmacol Rev 2005;57:173-185.

Kalinichev M, Easterling KW, Plotsky PM, Holtzman SG. Long-lasting changes in stress-induced corticosterone response and anxiety-like behaviors as a consequence of neonatal maternal separation in LongEvans rats. Pharmacol Biochem Behav 2002;73:131-140.

Lee JM, Park JM, Song MK, Oh YJ, Kim CJ, Kim YJ. The ameliorative effects of exercise on cognitive impairment and white matter injury from blood-brain barrier disruption induced by chronic cerebral hypoperfusion in adolescent rats. Neurosci Lett 2017;638:83-89.

Najjar S, Pearlman DM, Alper K, Najjar A, Devinsky O. Neuroinflammation and psychiatric illness. J Neuroinflammation 2013;10:43. 
Park SS, Shin MS, Park HS, Kim TW, Kim CJ, Lim BV. Treadmill exercise ameliorates nicotine withdrawal-induced symptoms. J Exerc Rehabil 2019;15:383-391.

Pittenger C, Duman RS. Stress, depression, and neuroplasticity: a convergence of mechanisms. Neuropsychopharmacology 2008;33:88-109.

Srikanth S, Nagaraja AV, Ratnavalli E. Neuropsychiatric symptoms in dementia-frequency, relationship to dementia severity and comparison in Alzheimer's disease, vascular dementia and frontotemporal dementia. J Neurol Sci 2005;236:43-48.
Strawbridge WJ, Deleger S, Roberts RE, Kaplan GA. Physical activity reduces the risk of subsequent depression for older adults. Am J Epidemiol 2002;156:328-334.

Teper E, O'Brien JT. Vascular factors and depression. Int J Geriatr Psychiatry 2008;23:993-1000.

Yang Y, Rosenberg GA. Blood-brain barrier breakdown in acute and chronic cerebrovascular disease. Stroke 2011;42:3323-3328.

Zlokovic BV. Neurodegeneration and the neurovascular unit. Nat Med 2010;16:1370-1371. 